\title{
MAGNETOHYDRODYNAMIC SIMULATION OF A STREAMER BESIDE A REALISTIC CORONAL HOLE
}

\author{
S. T. SUESS \\ Space Science Laboratory, NASA Marshall Space Flight Center \\ Huntsuille, Alabama 35812 U.S.A. \\ S. T. WU, A.-H. WANG \\ Center for Space Plasma and Aeronomic Research \\ The University of Alabama in Huntsville, Huntsville, Alabama 35899 \\ and \\ G. POLETTO \\ Osservatorio di Arcetri, Firenze, Italy
}

\begin{abstract}
Existing models of coronal streamers establish their credibility and act as the initial state for transients. The models have produced satisfactory streamer simulations, but unsatisfactory coronal hole simulations. This is a consequence of the character of the models and the boundary conditions. The models all have higher densities in the magnetically open regions than occur in coronal holes (Noci, et al., 1993).
\end{abstract}

Key words: Streamers - Coronal Holes - MHD Simulations

\section{Introduction}

An MHD numerical simulation of coronal streamers was described at the last SOHO workshop (Suess, 1992). That model used constant temperature and density at its base. The consequence was that the density in the magnetically open region was too large compared to what is expected in coronal holes. That problem is addressed here by using a varying temperature and density at the base. The reduced density in the coronal hole should improve simulation of SOHO observations of scattered light in the corona (Noci, et al., 1993).

\section{The Numerical Model and Results}

The model is for axisymmetric, polytropic, time-dependent, ideal magnetohydrodynamic flow and is computed in a plane containing the axis of the magnetic field. Results are given between 1.0 and $7.0 R_{\odot}$, although the solution extends to $15.0 R_{\odot}$. There are 20 grid points between the pole and equator and 37 grid points, on a varying grid, from the base to $15 R_{\odot}$. A solution is produced by a relaxation in time from an essentially arbitrary initial state. Here, the initial state is a vacuum dipole field superimposed on a Parker-type solar wind solution defined by the prescribed variation in tem-

Space Science Reviews 70, 295-298.

(1) 1994 Kluwer Academic Publishers. Printed in the Netherlands. 

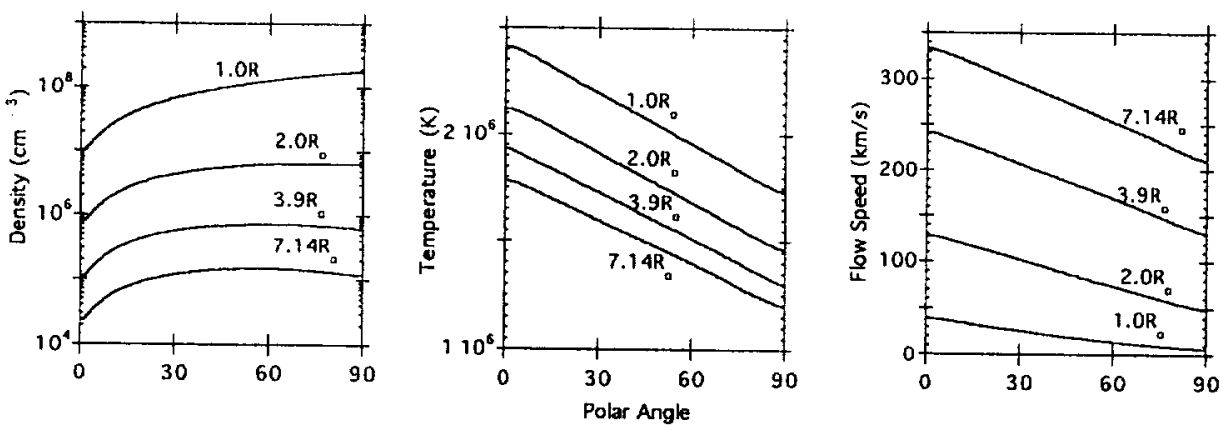

Fig. 1. The density, temperature, and flow speed at $t=0$.

perature and density at the base. The polytropic index is 1.05 . This model is described in detail by Suess (1992) and Wang, et al. $(1992,1993)$.

Figure 1 shows the initial profiles $(t=0)$ of temperature, density, and flow speed versus polar angle at $1.0,2.0,3.9$, and $7.1 R_{\odot}$. The plasma $\beta$, at the coronal base, is 3.5 at the equator and 0.06 at the pole, meaning field strengths of $0.833 \mathrm{G}$ and $1.67 \mathrm{G}$, respectively. The base densities at the equator and pole are $2 \times 10^{8}$ and $10^{7} \mathrm{~cm}^{-3}$, while the corresponding temperatures are $1.74 \times 10^{6}$ and $2.42 \times 10^{6} \mathrm{~K}$. The values between these points vary linearly. The polar base density must be low to obtain proper densities at higher levels in the coronal hole, while the temperature must be higher in the hole than in the streamer to obtain high flow speeds in the hole. No other choice is possible in a polytropic model. However, this 'effective temperature' is not a true temperature; it reflects extended acceleration and heating of the solar wind that is known to exist in coronal holes.

Figure 2 shows the magnetic field after 20 hours of relaxation in physical time, or five Alfvén times, in comparison to the initial vacuum dipole field (left panel) and to the equivalent steady state magnetic field topology with constant base temperature and density (center and right panels). The constant temperature and density used were $1.8 \times 10^{6}$ degrees and $2.25 \times 10^{8}$ $\mathrm{cm}^{-3}$, and the magnetic field strength at the equator was $1.67 \mathrm{G}(\beta=1)$ (Wang, et al., 1993). Field line footpoints lie at 10 degree intervals. It can thus be seen that fieldlines equatorward of somewhere between 40 and 50 degrees are closed. The center and right panels show that the low density and high flow speeds that occur in the present model lead to fieldlines lying systematically poleward of the case with the constant boundary conditions. This is a natural consequence of the higher energy in the flow field.

The dramatic difference introduced by varying the temperature and the density at the base is shown in Figure 3, whose format is identical to that in Figure 1. The results for constant boundary values are shown as dashed lines. The left panel shows that at 2.0 and $3.9 R_{\odot}$, the density at the center of the coronal hole is an order of magnitude less than with constant boundary 

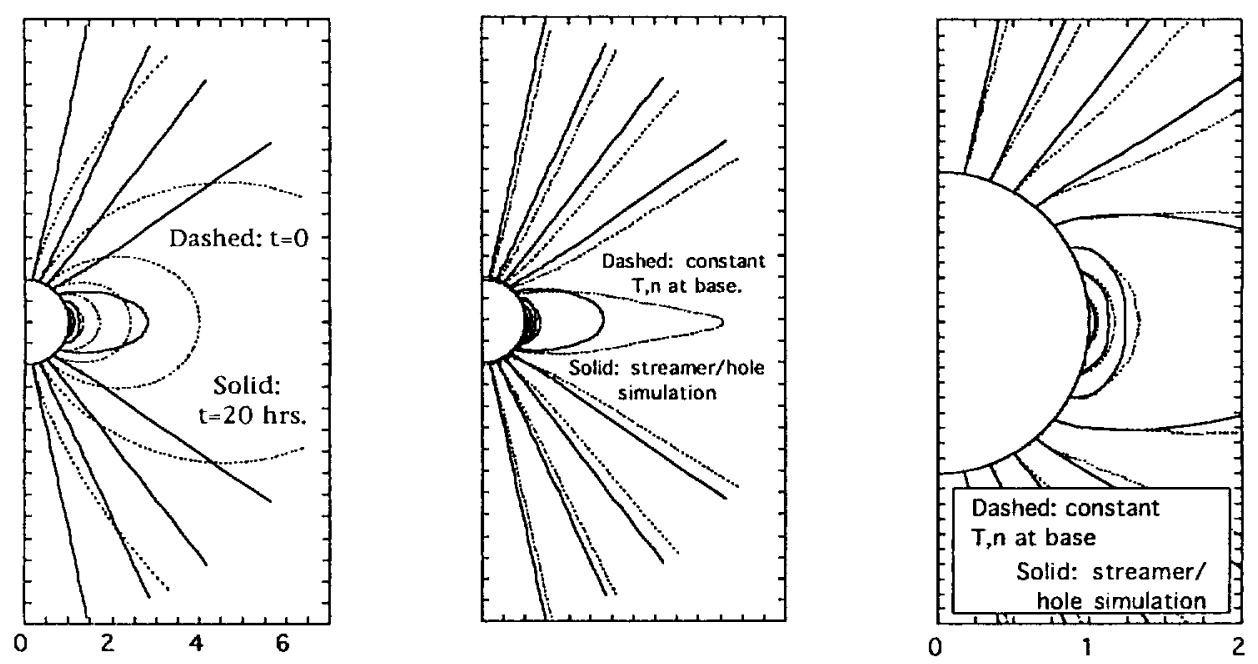

Fig. 2. Fieldline geometry for variable and constant boundary values.

values. The flow speed, in the right panel, is correspondingly much larger, reaching almost $250 \mathrm{~km} / \mathrm{s}$ at the center of the hole at $3.9 R_{\odot}$. The temperature varies little with height because of the polytropic index being 1.05 .

The low density in the coronal hole is mainly due to the low density at the base of the hole - the increase in flow speed only accounts for about a factor of two reduction. The limitation is that the polytrope has a large flow speed at $1.0 R_{\odot}$ at the base of the coronal hole $-40 \mathrm{~km} / \mathrm{s}$. This is an order of magnitude larger than expected at this level. The missing acceleration is not represented in the polytrope model and it is impossible to achieve both a low flow speed at the base and a high flow speed at $1.1-1.2 R_{\odot}$. It is a limitation also inherent in thermal conduction models in the absence of energy and momentum sources low in the corona.

The overall effect of lowering the density in the coronal hole is seen using the polar gray-scale plot of the logarithm of the number density shown in Figure 4. The left panel shows the hole/streamer with overlays of temperature contours, flow speed contours, and magnetic field lines. The right panel shows a corresponding plot for constant temperature and density at the base - the case shown by the dashed lines in Figure 3. The solid contours on the left half of the panels are flow speed (Min=0, Max $=350 \mathrm{~km} / \mathrm{s}, 50 \mathrm{~km} / \mathrm{s}$ contour levels). The dashed contours on the right half of the panels are temperature $\left(\mathrm{Min}=1 \times 10^{6}, \mathrm{Max}=2 \times 10^{6}, 2 \times 10^{5} \mathrm{~K}\right.$ contour levels). The solid lines on the right sides of the panels are magnetic field lines. Note that the density distribution on the right is far more spherically symmetric than on the left. Also, the temperature notch above the streamer is much narrower in the hole/streamer simulation. Finally, the density follows the contours of 

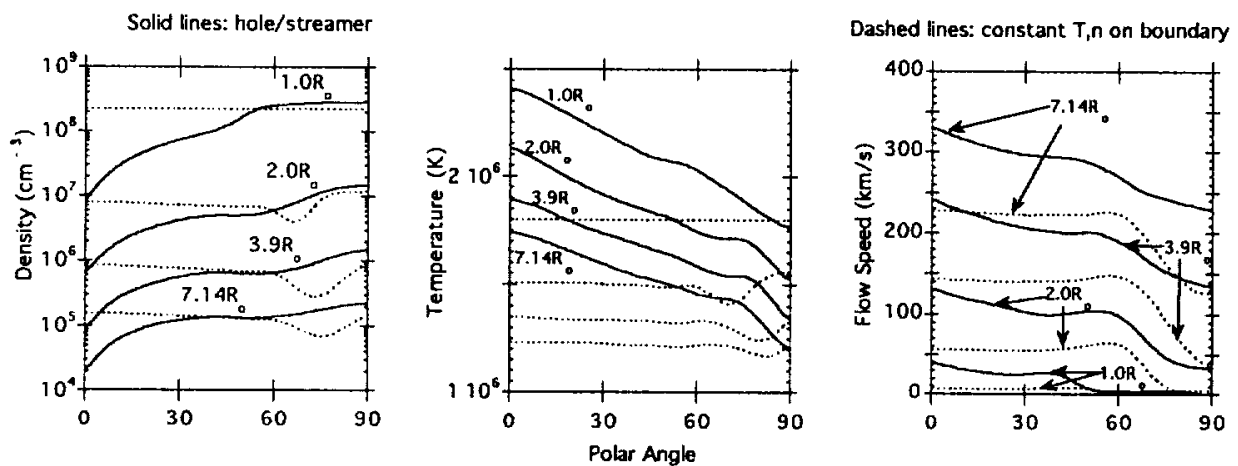

Fig. 3. The density, temperature, and flow speed at $t=20 \mathrm{hrs}$.
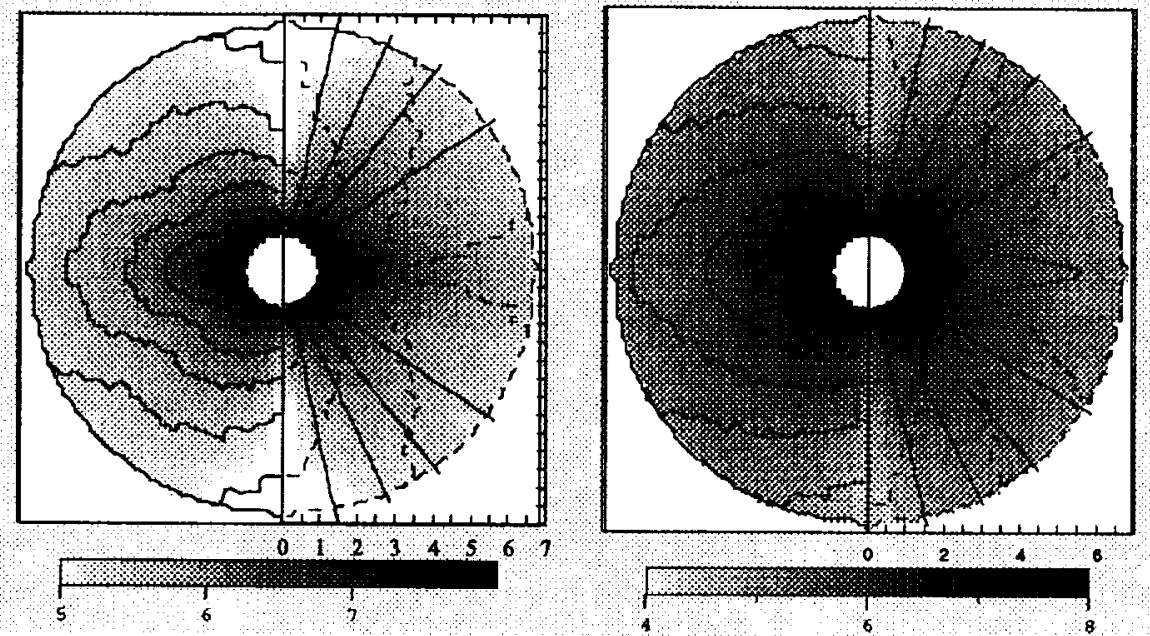

Fig. 4. Left: Coronal hole/streamer simulation. Right: Constant $\mathrm{T}, \mathrm{n}$ on boundary.

velocity better than the magnetic field lines. This reflects the control flow speed exerts on density in the corona - it is not a hydrostatic medium.

\section{References}

Noci, G., Poletto, G., Suess, S. T., Wang, A.-H., and Wu, S. T.: 1993, 'Ly- $\alpha$ Intensity in Coronal Streamers', Solar Phys. 147, 73

Parker, E. N.: 1963, Interplanetary Dynamical Processes, Interscience: New York

Suess, S. T.: 1992, 'Domingo, V., Poland, A., and Mariska, J.' in Modeling Solar Coronal Streamers, ed(s)., Coronal Streamers, Coronal Loops, and Coronal and Solar Wind Composition, SP-348, European Space Agency: Noordwijk, 63

Wang, A.-H., Wu, S. 'T., Suess, S. T., and Poletto, G.: 1992, 'Marsch, E., and Schwenn, R.' in A Two-Dimensional MHD Global Coronal Model: Steady-State Streamers, ed(s)., Solar Wind Seven, Pergamon Press: Oxford, 311

Wang, A.-H., Wu, S. T., Suess, S. T., and Poletto, G.: 1993, 'A Two-Dimensional MHD Global Coronal Model: Steady-State Streamers', Solar Phys. 147, 55 